\title{
Entropy-Based Localization of Textured Regions
}

\author{
Liliana Lo Presti and Marco La Cascia \\ University of Palermo \\ lopresti@dinfo.unipa.it
}

\begin{abstract}
Appearance description is a relevant field in computer vision that enables object recognition in domains as re-identification, retrieval and classification. Important cues to describe appearance are colors and textures. However, in real cases, texture detection is challenging due to occlusions and to deformations of the clothing while person's pose changes. Moreover, in some cases, the processed images have a low resolution and methods at the state of the art for texture analysis are not appropriate.

In this paper, we deal with the problem of localizing real textures for clothing description purposes, such as stripes and/or complex patterns. Our method uses the entropy of primitive distribution to measure if a texture is present in a region and applies a quad-tree method for texture segmentation.

We performed experiments on a publicly available dataset and compared to a method at the state of the art 16. Our experiments showed our method has satisfactory performance.
\end{abstract}

\section{Introduction}

In many applications, it is required a proper object description to enable recognition and/or classification. When the object is mainly a person, such description is related to the appearance and can be used to solve the people re-identification problem, very common in domain such as surveillance. There are many cues that can be used to perform re-identification; an approach could be to focus on the face and use facial features. However, this approach requires the face is adequately visible, and this is not the general case. Moreover, face descriptors are generally affected by pose and illumination changes so that the re-identification should be performed by using also other cues, i.e colors and textures in clothes.

Indeed, in case of people re-identification, clothing has an important role particularly when it presents some evident texture; e.g. "a person wearing a shirt with white and red stripes". Intuitively, an appearance description aiming to capture such properties would be more discriminative than a simple bag of words [] color description.

In the following we describe a method to discover "salient" structured areas in an image of a person that can be interpreted as "texture" characterizing person's clothing. Persons' clothing can be described by their colors and characteristics such as stripes, text and, broadly speaking, textures. To describe these kinds of 
properties, features robust to illumination changes should be used. For detecting texture we employ edge-based primitives, as they tend to be invariant to illumination. We use an approach inspired in some respects by the method of Kadir and Brady 15 for salient point detection. As we will explain later, we use an entropy based approach for detecting both the scale and the area of the texture in the object instance. In the method of Kadir and Brady, a strong response was obtained for textured areas that would result in false salient points; what was a limitation of their method is an advantage: for detecting interesting regions on a person's clothing that can ease object instance discrimination, the saliency measure is helpful, in the sense that a texture can be interpreted as a salient region; for example, considering a flat region, that is an area where no texture is present, a textured area would be salient inside this region.

The plan of the paper is as follows. In Section 2 we present related works and discuss important applications for the proposed method. In Sections 3 and 4, we present our method and discuss implementation details. In Sections 5 we present experimental results we got on a publicly available dataset and comparison to a method at the state of the art [16]. Finally, in Section 6 we present conclusions and future directions for our work.

\section{Related Works}

Appearance descriptors have an important role in establishing correspondences in multi-camera system to perform consistent labeling [6]. In [23], each object is represented as a "bag-of-visterms" where the visual words are local features. A model is created for each individual detected in the site. Descriptors consist of 128-dimensional SIFT vectors that are quantized to form visual words using a predefined vocabulary. In [18, appearance is modeled as bag of words in which a latent structure of features is recovered. A latent Dirichlet allocation (LDA) machine is used to describe appearance and discover correspondences between persons' instances.

The person re-identification task is not restricted just to video-surveillance systems but it is a recurrent problem in multimedia database management. A particular case is, for example, photo collection organization. In such application, Content Based Image Retrieval (CBIR) techniques and face features can be integrated in a probabilistic framework to define clusters of photos in order to ease browsing the collection [1]. In some works [5919], once the face is detected, the region under the face is used to compute information about the clothing of the person. In [21], face and clothing information are used for finding persons in many photos. First, a hierarchical clustering method is used for finding the set of persons whose face was detected in the photo sequence. Then, a clothing model is estimated for each person and used to recover mis-detected person's instances.

There are other applications for texture detection and recognition in clothing. Recently, in [24] a new method for clothes matching to help blind or color blind 
people has been presented. The method handles clothes with multiple colors and complex patterns by using both color and texture information.

In [8], the focus is on learning attributes, which are visual qualities of objects, such as red, striped, or spotted. To minimize the human effort needed to learn an attribute, they train models from web search engines. Once a model is learnt, it is capable of recognizing the attribute and determine its spatial extent in novel images. However, it seems impractical to enumerate all the possible kinds of textures that can arise in images. We believe, instead, the problem should be addressed at a lower visual level.

Texture detection and description are relevant problem, and a huge literature exists on the subject. Some works concern about texture detection for object segmentation [425] and classification [20. A common problem when working with texture is the scale selection 17/3. A texture can be detected and described by local properties and a proper neighborhood must be chosen. In particular, in [13] an approach based on the entropy of local patches is combined with a measure of the difference between neighboring patches to determine the best textel size for the texture.

In [16], a method to detect and localize instances of repeated elements in a photo is presented. Such elements may represent texels and the method is meant to detect repeated elements such as stripes in person's clothing. In this work, we consider a similar application. We use a multi-scale approach to find the best scale at which a texture can be detected. Texture is localized by using the entropy of some primitive and is segmented by a quad-tree strategy.

\section{Problem Definition}

Many definitions have been proposed for texture: Haralick 12] defines a texture as an organized area for which it exists a spatial distribution of a "primitive" defining some sort of ordered structure 22]. For Gibson, a texture is a characteristic based on which an area can appear as homogeneous [10].

The main goal in this work is to detect a predominant and well visible texture - for example, text or stripes on clothing - that can be used for describing the person appearance. Methods in literature, as for example 11, are not completely suitable for our application as they are generally conceived for high resolution images; moreover, generally objects are moving and undergoing articulated deformations so that fine details are not generally distinguishable and only rough texture can be discriminated.

Many methods for texture detection consider properties about the pixel intensity [13] or try to recognize specific patterns that can arise. However, the kind of textures we are interested in can be generally detected by exploring color organization with particular attention to their structure repeated over the space. As also proposed in other works 4], this structure can be highlighted, for example, by the edges in the image; therefore, an analysis of the detected edges can put in evidence some properties of the texture itself. Other properties can be explored 
too, as in the case of Law's energy measures where the basic features are: the average gray level, edges, spots, ripples and waves [2].

To segment a textured area, we need to measure how much a certain pixel belongs to a texture. This problem is strongly connected to the ability to detect the texel and the natural scale of a texture [22. For natural scale we mean the smallest size of the structure (texel) that can be spatially replicated to reproduce the texture itself [22]2].

In our approach, we use the entropy of a certain primitive to automatically detect the scale to use for segmenting the texture; then we use a split and merge approach for localizing and segmenting the texture.

\section{Texture Detection}

Detecting a texture requires reference to local properties measured in a suitable neighborhood. The size of this neighborhood is related - but not necessary equal - to the natural scale of the texture. In our approach, the scale of the texture is related to the size of the neighborhood that permits to have the best texture detection. To measure the presence of a texture, it makes sense to look at the local disorder measured for each pixel in an appropriate primitive space with respect to flat and ordered area. The most natural way is to use the entropy of these primitives; intuitively, the entropy will be maximum in textured areas. Other approaches in literature use the entropy for detecting the texture, as for example in [13, where the entropy is combined with other measures to detect textured area based on pixel intensities.

Given the probability distribution $P$ for a set of $N$ primitives, the entropy $E$ is defined as:

$$
E=\frac{1}{N} \sum_{i=1}^{N} P_{i} \cdot \log P_{i}
$$

Algorithm 1 summarizes the general framework we used for detecting pixel candidates to belong to a texture. In general, for each possible scale, we compute pixel per pixel the local entropy by using the statistic of the selected set of primitives in a suitable neighborhood. Points belonging to a texture have a very high entropy that decreases when the area becomes more and more flat. To segment the textured area, we take into account the spatial distribution of the pixels in the image: pixels near and with high entropy are much more probable to belong to a textured region.

\subsection{Primitives for Texture Detection}

The method described until now is quite general and applicable to different kinds of features as, for example, Gabor filters, Law's energy measures or features based on Local Binary Pattern [20. In this paper we use as primitive the orientation of the edges, even if any other primitive could be used. 

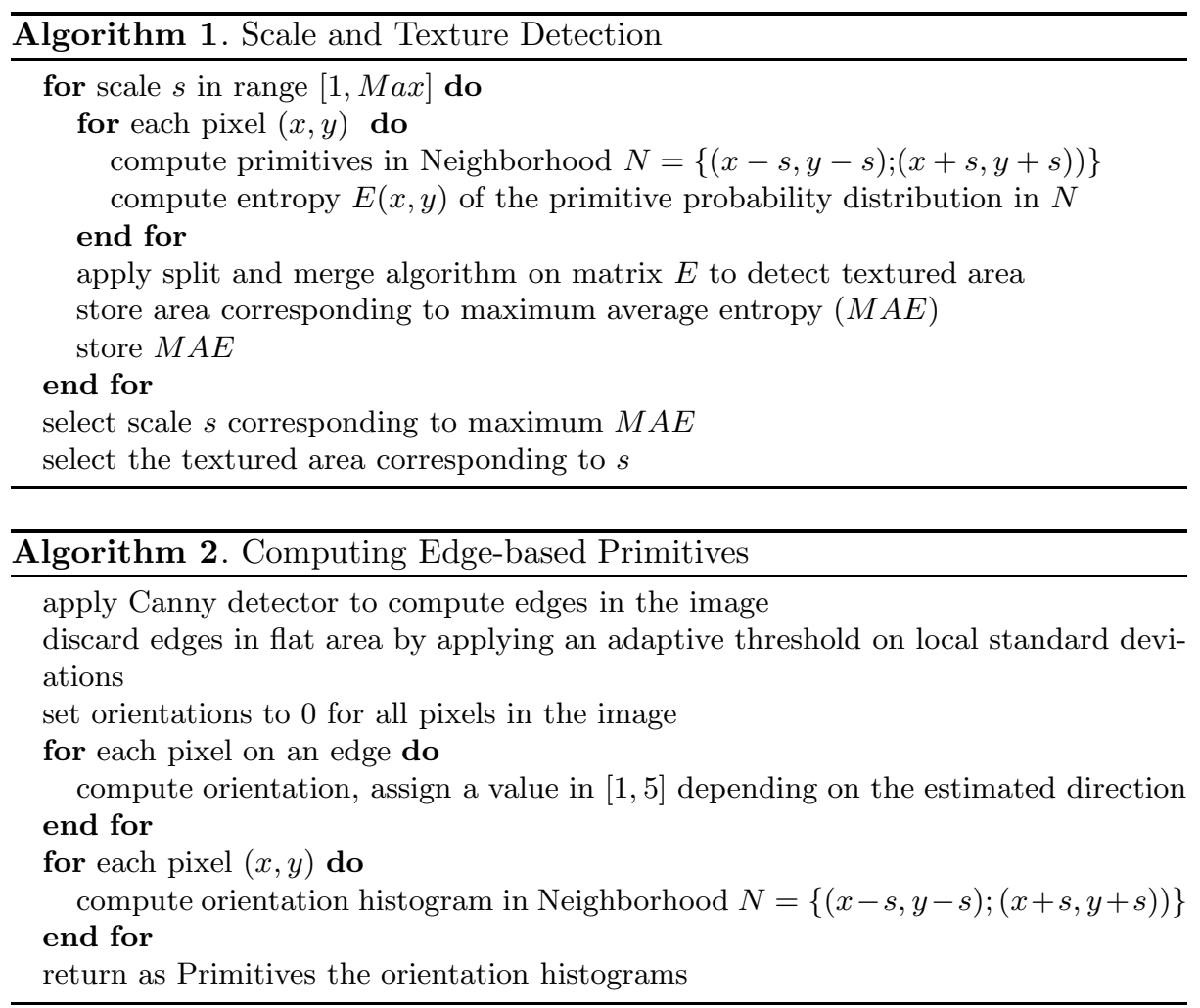

As we already said, a texture can be detected by analyzing properties of the detected edges. A texture is characterized by the presence of an organization/structure that can be detected considering the orientations of its edges. For each pixel on an edge we compute the orientation and then we quantize it so to consider only 4 predominant orientations: horizontal, vertical and obliques (that is $\pm 45 \mathrm{deg}$ ). Orientations are computed by using the gradient components of the gray level image. Algorithm 2 reports the pseudo-code for the extraction of this kind of primitives. Not all the detected edges belong to a texture but some of them are in flat areas, in correspondence to wrinkles in the clothing or self-occlusions of the person. To improve the edge detection, we filter them to remove edges in areas with homogeneous colors. The remaining edges are then used to compute the statistic of the orientations in a local neighborhood. The size of this neighborhood is related to the scale $s$ the texture is detected to. Given a neighborhood and the orientations of the edges in it, we computed an histogram of 5 bins: 1 for counting pixels in flat areas, and 4 for considering the orientations of the edges within the neighborhood. Based on this histogram, for each pixel we computed the entropy associated to the pixel within a neighborhood of size $2 \cdot s$ (the scale). 


\subsection{Texture Segmentation}

We segment the textures by a split and merge approach to construct a quad-tree. For splitting, we consider how much homogeneous each quad is and we used the local standard deviation of the entropy assigned to each pixel as metric. The minimum quad size during decomposition has been set to $8 \times 8$ pixels, while the threshold on the standard deviation has been set to 0.1 .

For the merging step, instead, we merge all the neighbor quad regions that have similar entropies. In this case, the threshold has been set to 0.3 . The success of the merging step depends strongly on the order with which the quads are merged together. We start the merging phase from the quad presenting the highest entropy.

For selecting the correct scale, we considered the neighborhood size that gives the best texture representation, that is we choose the scale corresponding to the maximum average entropy in the detected area. At the selected scale, we consider the region with the highest entropy, and we apply a threshold $\tau_{E}$ to determine if such region can be classified as texture.

Fig. 1a shows an example with an artificial texture where each quad is 10 pixel large (so that the natural scale should be 20 pixels). Figure $1 \mathrm{~b}$ shows how the expected entropy in the textured area varies with respect to the scale. As the figure shows, the entropy has a periodic trend depending on the scale of the texture. The selected scale is chosen to be the first peak.

In real case images, however, the trend of the entropy is not so regularly periodic because of the noise in the images, and the deformation to which primitives generally undergo due to different orientations and person's pose. In this case, we consider the scale corresponding to the maximum value of the entropy.
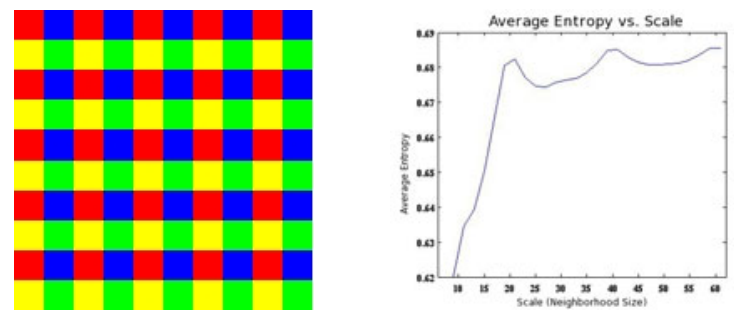

Fig. 1. Artificial texture and average entropy at different scales

\subsection{Implementation Details}

Fig. 2 shows two examples of texture detection on surveillance data. The goodness of the detection strongly depends on the operator used for the edge detection and the filtering step. In our implementation, we computed edges by considering the maximum response of a Canny detector applied to each color channel. Filtering was performed discarding all the edges in color flat area. This area were determined by looking at the maximum local standard deviation of the channel intensities in a 
neighborhood of size $5 \times 5$ pixels. We applied a local threshold to maintain all the edges having standard deviation in the $65 \%$ confidence interval. In our implementation, the threshold $\tau_{E}$ on the entropy has been set empirically to 0.4 .

Performance of the multi-scale approach has been considerably improved by using integral images when computing the histogram for each orientation. In our experiments, when a texture is detected, the corresponding scale changes according to the distance of the object from the camera; we noted that in sequences of frames, it is possible to track the scale itself. In this case, once a scale is detected, the corresponding value may be used as prior for the next frame to process so that not all the range of possible values for the scale is spanned but only those values in a neighborhood of the prior scale value, speeding up the computation.

We also note that the texture detector fails when there are too strong features in the image not related to a texture, for example when there are too evident wrinkles in clothing or a cluttered background, and the filtering step is not able to classify the area around the edge as homogeneous. Another case of failure arises, of course, in case the edge detector fails.
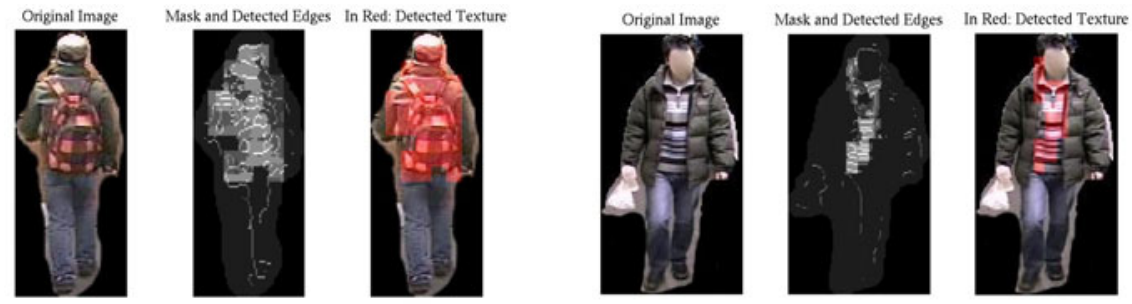

Fig. 2. Textures detected on surveillance data

\section{Evaluation}

We tested our method on a publicly available dataset 9 . To measure the performance, we randomly choose a subset of images. For each image, we detected the face and considered the region under the face as possible clothing region. We considered a subset of 50 persons. Of these images, 16 did not have any textured region in the clothing, while the remaining 34 had. For each image, we manually detected the region corresponding to the texture in the clothing (see fig. 33).
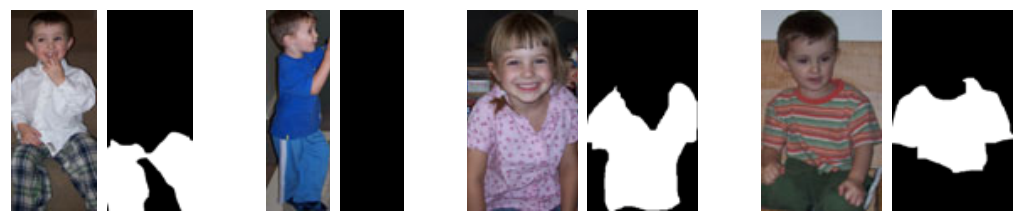

Fig. 3. Test images and corresponding manually segmented ground-truth 
As the detection method works on local properties, we measured the performance by dividing the image in sub-windows (blocks) of $30 \times 30$ pixels. For each block, we computed the balanced accuracy defined as:

$$
B A=\frac{\text { sensitivity }+ \text { specificity }}{2}=\frac{1}{2} \cdot \frac{T P}{T P+F N}+\frac{1}{2} \cdot \frac{T N}{T N+F P}
$$

where $T P, T N, F P$, and $F N$ are true positive, true negative, false positive and false negative respectively. This performance measure treats both classes (the positive and the negative ones) with equal importance [3], and it is particularly appropriated in our case as we want to measure the ability to correctly classify a pixel as belonging to a texture or not.

We considered correctly classified a sub-window for which the balanced accuracy was greater than a threshold $\tau_{a}$ and, for each image, we measured the ratio of the correctly classified blocks over the total number of sub-windows.

For comparison purposes, we tested our method against the one in 16. This method is conceived to detect and group repeated scene elements representing complex patterns or stripes from an image. First, the method detects interesting elements in the image by analyzing the structure tensor; such candidates are matched against their neighbors estimating the affine transform between them. The elements are grown and iteratively grouped together to form a distinctive unit (for more details, refer to [16]). In Fig. 4, the graph shows the curves of the average classification rate over the whole dataset for different values of the threshold $\tau_{a}$ for our method and for the one in [16. $\tau_{a}=1$ represents the ideal case the texture is always correctly detected and in such a case our method shows a classification rate of about $64 \%$ while the other method has a classification rate equals to $51.3 \%$. For a balanced accuracy of about $80 \%\left(\tau_{a}=0.8\right)$, our method shows a classification rate of $79.27 \%$ while the other method has a classification rate equals to $61.5 \%$. In comparison to [16], our method presents an higher specificity and showed to be more robust when detecting text and complex patterns. The method in [16] suffers from the fact that the scale is not automatically detected and it is unable to detect those regions where the texel size is large. On the contrary, in our method the scale is automatically detected by evaluating the entropy as already explained in section 4 .

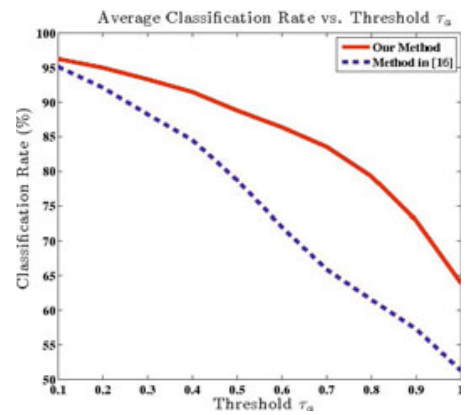

Fig. 4. Comparison between our method and the one in 16 


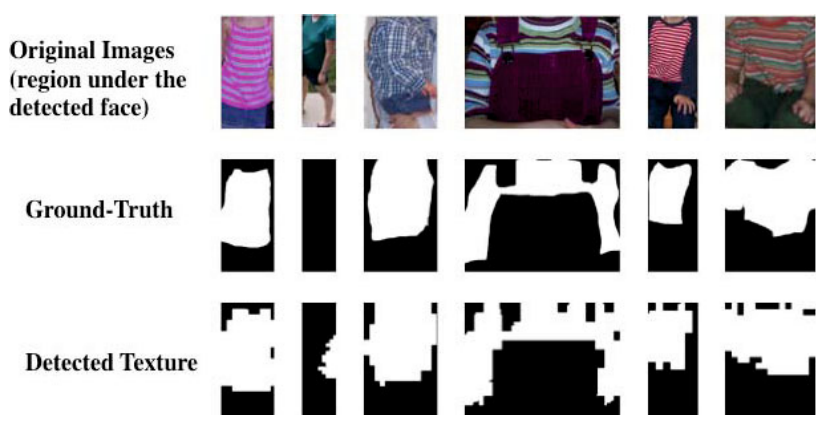

Fig. 5. Results by applying our method on the test images

Fig. 5 shows an example of results we got by applying our method. As the figure shows, the method can be sensitive to the background (see second image in the figure). However, this problem could be limited using proper algorithm to detect persons. In surveillance application, background suppression could be adopted. In photo collections, the method in [9] can be used to approximate the clothing region, then our method can be used to find the textured area.

\section{Conclusion and Future Works}

In this paper, we proposed a new method to detect textured area in clothes region. Our method computes the predominant orientation in a neighborhood for each pixel and uses entropy to capture the disorder associated to such a primitive distribution. A split and merge approach is then used to segment the textured area. Our method is able to capture 2D structures representing complex (i.e. text) or regular patterns (i.e. stripes) in clothing regions. We deal with the problem of selecting the scale of the texture automatically by adopting a multi-scale approach and using the entropy as measure of the goodness of such selection. In future works, we will study how the entropy distribution can be used also for description purposes and if it is possible to use it to perform classification. We will also study techniques for fusing color and texture information to enhance person re-identification by using probabilistic frameworks.

\section{References}

1. Ardizzone, E., La Cascia, M., Vella, F.: A novel approach to personal photo album representation and management. In: Proc. of Multimedia Content Access: Algorithms and systems II. IS\&T/SPIE Symp. on EI (2008)

2. Ballard, D.H., Brown, C.: Computer Vision. Prentice-Hall, Englewood Cliffs

3. Brodersen, K.H., Ong, C.S., Stephan, K.E., Buhmann, J.M.: The balanced accuracy and its posterior distribution. In: Proc. of Int. Conf. on Pattern Recognition (ICPR), pp. 3121-3124 (2010)

4. Carson, C., Belongie, S., Greenspan, H., Malik, J.: Blobworld: image segmentation using expectation-maximization and its application to image querying. Trans. on Pattern Analysis and Machine Intelligence 24(8), 1026-1038 (2002)

5. Cooray, S., O'Connor, N.E., Gurrin, C., Jones, G.J.F., O'Hare, N., Smeaton, A.F.: Identifying Person Re-Occurences For Personal Photo Management Applications. In: IET Int. Conf. on Visual Information Engineering (VIE), pp. 144-149 (2006) 
6. Doretto, G., Sebastian, T., Tu, P., Rittscher, J.: Appearance-based person reidentification in camera networks: problem overview and current approaches. Journal of Ambient Intelligence and Humanized Computing, 1-25 (2010)

7. Fei-Fei, L., Perona, P.: A Bayesian Hierarchical Model for Learning Natural Scene Categories. In: Proc. of Computer Vision and Pattern Recognition, pp. 524-531 (2005)

8. Ferrari, V., Zisserman, A.: Learning visual attributes. Advances in Neural Information Processing Systems (2008)

9. Gallagher, A.C., Chen, T.: Clothing cosegmentation for recognizing people. In: Proc. of. Computer Vision and Pattern Recognition (CVPR), pp. 1-8 (2008)

10. Gibson, J.J.: The Perception of the Visual World. Houghton Mifflin, Boston (1952)

11. Han, B., Yang, C., Duraiswami, R., Davis, L.: Bayesian Filtering and Integral Image for Visual Tracking. In: IEEE Proc. of Int. Workshop on Image Analysis for Multimedia Interactive Services, WIAMIS 2005 (2005)

12. Haralick, R.M.: Statistical and structural approaches to texture. In: Proc. IEEE (1979)

13. Hong, B.-W., Soatto, S., Ni, K., Chan, T.: The scale of a texture and its application to segmentation. In: Proc. of Int. Conf. on Computer Vision and Pattern Recognition (CVPR), pp. 1-8 (2008)

14. Huang, T., Russell, S.: Object Identification in a Bayesian Context. In: Proc. the Int. Joint Conf. on Artificial Intelligence (IJCAI), pp. 1276-1283 (1997)

15. Kadir, T., Brady, M.: Saliency, Scale and Image Description. Int. J. Comput. Vision 45, 83-105 (2001)

16. Leung, T., Malik, J.: Detecting, localizing and grouping repeated scene elements from an image. In: Buxton, B.F., Cipolla, R. (eds.) ECCV 1996. LNCS, vol. 1064, pp. 546-555. Springer, Heidelberg (1996)

17. Lindeberg, T.: Feature Detection with Automatic Scale Selection. Int. Journal of Computer Vision 30(2), 79-116 (1998)

18. Lo Presti, L., Sclaroff, S., La Cascia, M.: Object Matching in Distributed Video Surveillance Systems by LDA-Based Appearance Descriptors. In: Foggia, P., Sansone, C., Vento, M. (eds.) ICIAP 2009. LNCS, vol. 5716, pp. 547-557. Springer, Heidelberg (2009)

19. Lo Presti, L., Morana, M., La Cascia, M.: A Data Association Algorithm for People Re-Identification in Photo Sequences. In: IEEE Proc. of Int. Symposium on Multimedia (ISM), pp. 318-323 (2010)

20. Ojala, T., Pietikainen, M., Harwood, D.: A comparative study of texture measures with classification based on featured distributions. Pattern Recognition 29(1), 5159 (1996)

21. Sivic, J., Zitnick, C., Szeliski, R.: Finding people in repeated shots of the same scene. Proc. BMVC 3, 909-918 (2006)

22. Schwartz, J.: Studies in Visual Perception, IV - Homogeneous Textures, New York University

23. Teixeira, L.F., Corte-Real, L.: Video object matching across multiple independent views using local descriptors and adaptive learning. Pattern Recogn. Lett. 30(2), 157-167 (2009)

24. Tian, Y., Yuan, S.: Clothes matching for blind and color blind people. In: Proc. of Int. Conference on Computers helping people with special needs, pp. 324-331. Springer, Heidelberg (2010)

25. Voorhees, H., Poggio, T.: Computing texture boundaries from images. Nature 333(6171), 364-367 (1988) 